\title{
BMJ Open Comparison of cardiovascular disease risk factors, assessment and management in men and women, including consideration of absolute risk: a nationally representative cross- sectional study
}

\author{
Emily Banks (D) , Jennifer Welsh (D), Grace Joshy (D), Melonie Martin (D), \\ Ellie Paige (10), Rosemary J Korda (1)
}

To cite: Banks E, Welsh J, Joshy G, et al. Comparison of cardiovascular disease risk factors, assessment and management in men and women, including consideration of absolute risk: a nationally representative crosssectional study. BMJ Open 2020;10:e038761. doi:10.1136/ bmjopen-2020-038761

- Additional material is published online only. To view please visit the journal online (http://dx.doi.org/10.1136/ bmjopen-2020-038761)

Received 24 March 2020 Revised 15 0ctober 2020 Accepted 13 November 2020

Check for updates

(c) Author(s) (or their employer(s)) 2020. Re-use permitted under CC BY-NC. No commercial re-use. See rights and permissions. Published by BMJ.

National Centre for Epidemiology and Population Health, College of Health and Medicine, Australian National University, Canberra, Australian Capital Territory, Australia

Correspondence to Professor Emily Banks; emily.banks@anu.edu.au

\section{ABSTRACT}

Objectives Cardiovascular disease (CVD) is highly preventable and optimal treatments based on absolute risk can halve risk of future events. Compared with women, men have higher risks of developing CVD. However, women can experience suboptimal treatment. We aimed to quantify sex differences in CVD risk, assessment and treatment in Australian adults.

Design, participants, setting Cross-sectional analysis of nationally representative data from interview, physical measures, medication review and blood and urine samples, from 2011 to 2012 Australian Health Survey participants aged $45-74(n=11518)$.

Outcome measures CVD risk factors, absolute 5year risk of a primary CVD event, blood pressure and cholesterol assessment in the previous 2 and 5 years and use of recommended CVD preventive medications were compared using Poisson regression to estimate ageadjusted male versus female prevalence ratios (PRs).

Results Women had a generally more favourable CVD risk factor profile than men, including lower: current smoking prevalence (women $=14.5 \%$; men $=18.4 \%$, $\mathrm{PR}=0.78,95 \% \mathrm{Cl}=0.70$ to 0.88 ); body mass index (women $($ mean $)=28.3 \mathrm{~kg} / \mathrm{m}^{2} ;$ men (mean) $=28.8 \mathrm{~kg} / \mathrm{m}^{2}, p<0.01$; systolic and diastolic blood pressure (systolic: women (mean) $=127.1 \mathrm{~mm} \mathrm{Hg}$; men (mean) $=130.5 \mathrm{~mm} \mathrm{Hg}$, $\mathrm{p}<0.001$ ); blood glucose (women (mean) $=5.2 \mathrm{mmol} / \mathrm{L}$; men (mean) $=5.5 \mathrm{mmo} / \mathrm{L}$ ); diabetes prevalence (women $=6.8 \%$; men $=12.5 \%, \mathrm{PR}=0.55,95 \% \mathrm{Cl}=0.44$ to 0.67 ); prior CVD (women=7.9\%; men=11.3\%) and absolute primary CVD risk (absolute 5-year CVD risk $>15 \%$ : women $=6.6 \%, 95 \% \mathrm{Cl}=5.4$ to 7.8 ; $\mathrm{men}=15.4 \%$, $95 \% \mathrm{Cl}=13.9 \%$ to $16.9 \%$ ). Compared with men, women had higher low-density lipoprotein, high-density lipoprotein and total cholesterol and sedentary behaviour and lower physical activity. Blood pressure and cholesterol assessment were common in both sexes. Among those at high absolute risk, age-adjusted proportions receiving recommended CVD medications were low, without sex differences (women $=21.3 \%$; men $=23.8 \%, P R=0.93$, $95 \% \mathrm{Cl}=0.49$ to 1.78 ). Fewer women than men with
Strengths and limitations of this study

- This paper is the first to compare men and women comprehensively in terms of data on cardiovascular disease (CVD) risk factors, absolute risk, assessment and treatment.

- We used nationally representative data that included detailed biological and behavioural CVD risk factors, measured directly at interview or with fasting blood and urine samples.

- Data on behavioural or lifestyle interventions to manage CVD risk were not available, nor were data on the reasons for a lack of treatment for people with existing CVD or with high absolute risk.

prior atherosclerotic CVD were receiving recommended treatment (women $=21.8 \%$, men $=41.4 \%, P R=0.55,95 \%$ $\mathrm{Cl}=0.31$ to 0.96 ).

Conclusion Women have a more favourable CVD risk factor profile than men. Preventive treatment is uncommon and women with prior atherosclerotic CVD are around half as likely as men to be receiving recommended treatment.

\section{INTRODUCTION}

Cardiovascular disease (CVD) is a leading cause of morbidity and mortality worldwide, with 366 million disability-adjusted life years attributed to CVD in $2017^{1}$ and an estimated 17646000 deaths in 2016. ${ }^{2}$ An estimated 1.2 million adults in Australia are living with CVD. ${ }^{3}$ Management of CVD and its risk factors, including using an absolute risk approach, are known to improve outcomes, including preventing future CVD events such as myocardial infarction, stroke and death from CVD. ${ }^{4-6}$

Compared with women, for a given age, men have higher risks of developing virtually 
all types of CVDs and of dying from CVD. ${ }^{7}$ While the reasons for these differences have not been quantified precisely, their greater burden of CVD risk factors such as smoking, high blood pressure and diabetes is likely to contribute. ${ }^{89}$ Current evidence also indicates that women can experience delays in treatment, less intensive treatment for CVD and less risk assessment, compared with men. ${ }^{1011}$

Given the highly preventable nature of CVD, evidence regarding the appropriate targeting of interventions, including those aimed at reducing sex disparities, is essential to ongoing efforts to reduce its impact. Although there is a growing body of evidence on sex differences in CVD risk factors and management, comprehensive representative population-level evidence is limited, including in relation to absolute CVD risk and management. The aims of this study are to quantify differences between Australian men and women in their profiles of (1) behavioural and biomedical CVD risk factors, (2) 5-year absolute CVD risk, (3) blood pressure and cholesterol assessment and (4) guideline-recommended use of CVD medications.

\section{METHODS}

\section{Study population}

We used interview-based 'core content' data from adults aged between 45 and 74 years who participated in 20112012 Australian Health Survey (AHS), ${ }^{12}$ a nationally representative survey of private dwellings (excluding very remote areas of Australia and discrete Aboriginal and Torres Strait Islander communities) covering about 97\% of people living in Australia. ${ }^{13}$ The AHS comprises of three substudies: the National Health Survey (NHS), the National Nutrition and Physical Activity Survey (NNPAS) and the National Health Measure Survey (NHMS). Core content data, which included common data items on household characteristics, physical measures (eg, height, weight, blood pressure), smoking status and health conditions, were collected as part of the NHS and the NNPAS. The NHMS, designed to measure biomarkers for chronic disease and nutritional status, included fasting and non-fasting blood and urine tests collected by qualified phlebotomists at collection clinics or via a home visit. ${ }^{13}$

NHS and NNPAS participants were sampled using a stratified multistage area sample of private dwellings. Within dwellings, one adult (aged 18 years and older) and, if applicable, one child aged 0-17 years (NHS) or one child aged 2-17 years (NNPAS) were randomly sampled to take part in the study. All NHS and NNPAS participants aged 5 years and over were invited to take part in the NHMS.

\section{Patient and public involvement}

Not applicable. Patients were not involved in the development of this study.

\section{Measures}

Information on sociodemographic factors (age, country of birth, region of residence and highest level of education) and health behaviours (physical activity, smoking, alcohol intake) were self-reported during home-based interview. Height and weight (used to estimate body mass index (BMI)), waist circumference and blood pressure were measured directly during interviews. Fasting blood and urine samples were collected and assayed to measure haemoglobin A1c (HbAlc), fasting glucose, glomerular filtration rate, low-density lipoprotein (LDL), high-density lipoprotein (HDL) and total cholesterol, triglycerides and microalbuminuria. Respondents were considered to have diabetes if they had a fasting blood glucose of $\geq 7.0 \mathrm{mmol} / \mathrm{L}$ and $/$ or a HbAlc of $\geq 48 \mathrm{mmol} / \mathrm{L}$ and/or were taking medication for diabetes. ${ }^{6}$ Microalbuminuria was defined as albumin:creatinine of $\geq 2.5 \mathrm{mg}$ / mmol for men or $\geq 3.5 \mathrm{mg} / \mathrm{mmol}$ for women. ${ }^{6}$ Moderate to severe chronic kidney disease was defined as a glomerular filtration level of $<45 \mathrm{~mL} / \mathrm{min} / 1.73 \mathrm{~m}^{2} .{ }^{6}$

Participants were considered to have prior CVD if they self-reported ever being diagnosed with ischaemic heart disease, heart failure, other heart diseases, cerebrovascular disease, diseases of the arteries, arterioles and capillaries or current and long-term oedema. Prior atherosclerotic CVD was defined as ischaemic heart disease, cerebrovascular disease or disease of the arteries, arterioles and capillaries; finer subtyping was not possible with the available data. Absolute risk of a primary CVD event was calculated using the Australian National Vascular Disease Prevention Alliance algorithm. This algorithm combines clinical high-risk criteria and the Framingham risk equation to estimate 5-year absolute risk of a primary CVD event, grouped into low $(<10 \%$ risk), moderate $(10 \%-15 \%)$ or high $(>15 \%)$ absolute risk. ${ }^{6}$ Low risk corresponds to $<10 \%$ probability of CVD within the next 5 years; moderate risk corresponds to $10 \%-15 \%$ probability of CVD within the next 5 years and high risk corresponds to $>15 \%$ probability of CVD within the next 5 years.

Participants were asked whether they had their blood pressure measured in the previous 2 years and their cholesterol measured in the previous 5 years, based on the recommended minimum intervals. ${ }^{14}$

CVD medication use was assessed as part of a full medication review and coded according to the WHO Anatomical Therapeutic Chemical (ATC) classification system. ${ }^{15}$ Guidelines recommend that individuals at high absolute risk be treated with combined blood pressure-lowering and lipid-lowering medications ${ }^{6}$ and additionally with antithrombotic medication for those with prior atherosclerotic CVD. ${ }^{16}{ }^{17}$ ATC codes for ascertaining blood pressure-lowering medication were C02, C03, C07, C08 and C09; C10 for lipid-lowering medications and B01 for antithrombotic medications. ${ }^{15}$

\section{Statistical approach}

Sex differences in the distribution and prevalence of CVD risk factors in the Australian population were examined, 
with continuous risk factors plotted for men and women separately. Poisson regression with jackknife standard errors estimated the age-adjusted prevalence of each risk factor and absolute and relative sex differences in each risk factor. Prevalence ratios (PRs) were estimated directly from the Poisson regression coefficients and postestimation marginal effects were used to obtain prevalence differences (PDs).

We estimated the distribution of absolute CVD risk in the Australian population using data from NHMS participants, including those who were clinically determined to be at high primary risk and those with prior CVD. The proportion and number of Australian men and women with prior CVD and with low, moderate and high primary CVD risk were then estimated.

By level of absolute risk, we estimated the proportion and number of Australian men and women receiving blood pressure and cholesterol assessments in the previous 2 and 5 years, respectively, and the proportions taking CVD medications. Modified Poisson regression was used to estimate absolute and relative sex differences in the receipt of these assessments, and for those at high absolute risk or with prior atherosclerotic CVD, differences in taking medications. Models were sequentially adjusted, first for age and then additionally for region of residence, country of birth and highest level of education. Men were used as the reference group for all analyses.

There were no missing data on medication use. Those with missing data on behavioural and biomedical CVD risk factors and blood pressure and cholesterol assessments were excluded from the corresponding analyses. Missing data on covariates in adjusted models were coded as a separate category and included in the analysis. Weights, created by the Australian Bureau of Statistics
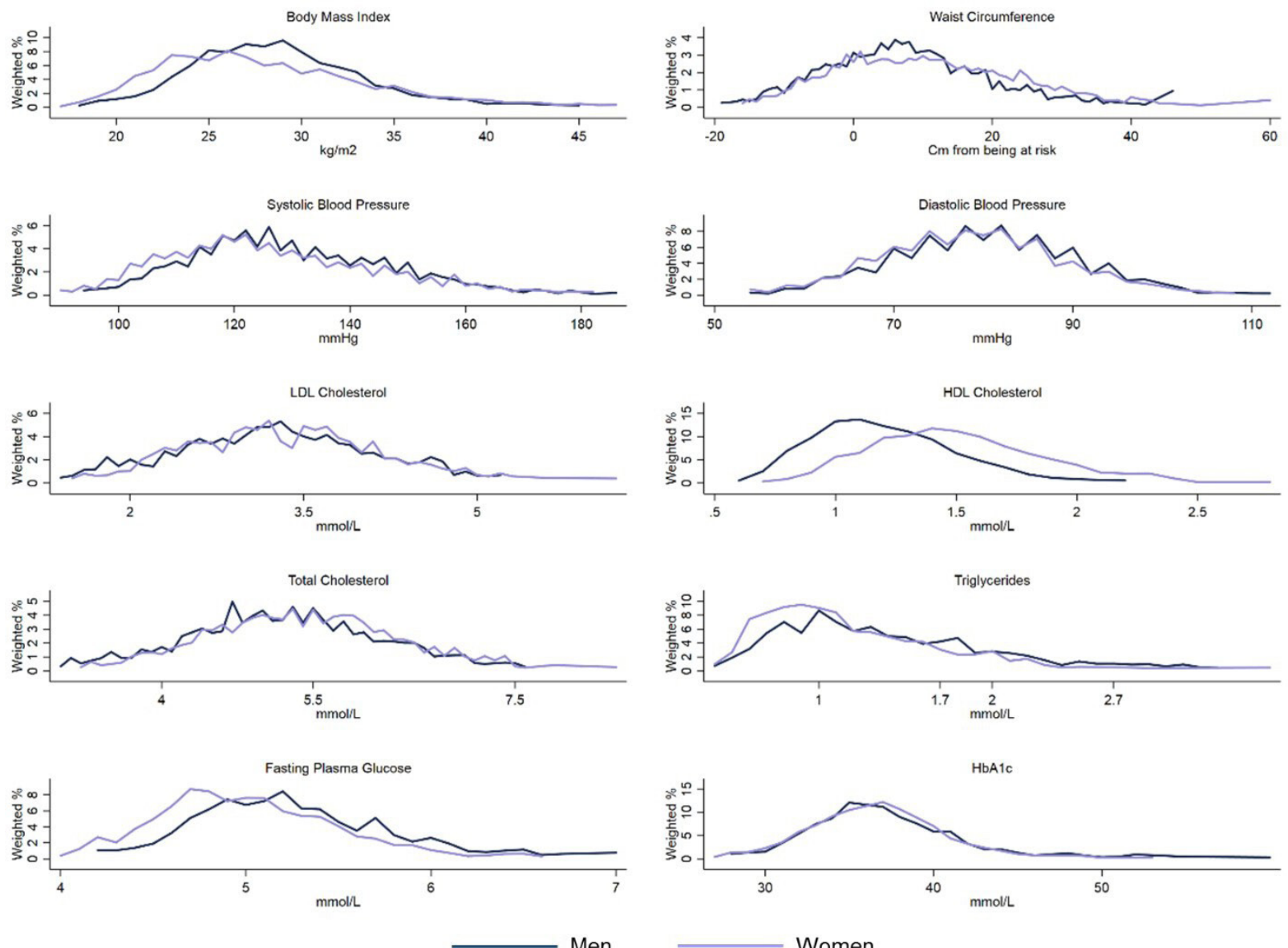

Men

Women

Figure 1 Distribution of CVD risk factors in the Australian population aged 45-74 years, by sex. BMI, waist circumference, systolic blood pressure and diastolic blood pressure were measured as part of the core content for the Australian Health Survey ( $n=11518$ ). Proportion of missing values: BMI: 15.9\%; waist circumference: $16.0 \%$; systolic blood pressure: $15.3 \%$; diastolic blood pressure: 15.3\%. LDL, HDL and total cholesterol, and triglycerides, fasting plasma glucose and HbA1c were measured as part of the National Health Measures Survey $(n=5253)$. Proportion of missing values: LDL cholesterol: $20.6 \%$; HDL: 0.7\%; total cholesterol 0.7\%; triglycerides: 19.4\%; fasting plasma glucose: $19.4 \%$; HbA1c: 0.9\%. All estimates have been weighted to be representative of the Australian population living in non-very remote areas. The $\mathrm{x}$-axis for waist circumference is estimated with the difference between waist circumference and the sex-specific cut points for an 'at risk' waist circumference ( $80 \mathrm{~cm}$ for women, $94 \mathrm{~cm}$ for men). Body mass index and waist circumference are rounded to the nearest whole number. Systolic and diastolic blood pressure are rounded to the nearest second number. Risk factor values with less than 10 respondents have been suppressed. BMI, body mass index; HbA1c, haemoglobinA1c; HDL, high-density lipoprotein; LDL, low-density lipoprotein. 
(ABS) and benchmarked to the estimated number of residents living in private dwellings in non-very remote areas of Australia, were applied to all analyses. ${ }^{18}$ The number of Australian adults receiving blood pressure and cholesterol assessments and the number using CVD medications were estimated by applying the weighted proportions to the Australian general population data. ${ }^{13}$ SEs were estimated using the delete-a-group Jackknife methods using 60 replicate weights provided by the ABS. Analyses were performed in the DataLab, with approval from the ABS, using Stata V.15.1.

\section{Supplementary analyses}

Supplementary analyses estimated risk factor and absolute risk distributions, PDs and PRs in men and women aged 18 years and over and in those aged 45-74 years without prior CVD. Finally, analyses of medication use restricted the diagnosis of prior CVD to ischaemic heart disease only.

\section{RESULTS}

Among study participants aged $45-74$ years, there were 11518 in the core content of the AHS and 5353 in the NHMS. Full information needed to estimate absolute CVD risk was available for 4833 participants (2210 men and 2623 women). The characteristics of the sample are presented in online supplemental table 1 (corresponding numbers for $\geq 18$ years: online supplemental table 2).

\section{CVD risk factors}

Compared with men, women had lower average BMI, waist circumference, systolic blood pressure, diastolic blood pressure, total: HDL cholesterol ratio, triglycerides, fasting plasma glucose and HbAlc; they had higher mean HDL, LDL and total cholesterol levels (figure 1, table 1). Overall, a lower proportion of women compared with men were overweight, current and former smokers and consumers of $>14$ standard drinks/week (figure 2). Diabetes and diabetes with microalbuminuria were less common among women than men. However, a higher proportion of women than men had an at-risk waist circumference, high total cholesterol and low physical activity. There were no differences observed between men and women in the prevalence of very high systolic blood pressure, high LDL cholesterol or chronic kidney disease.

Differences between CVD risk factors in men and women were similar when data were expanded to those aged $\geq 18$ years and when restricted to people without prior CVD, however, in adults aged $\geq 18$ years a smaller proportion of women than men had high LDL cholesterol levels (online supplemental figures S1 and S2 and table S3), (online supplemental figures S3 and S4 and table S4).

\section{5-year absolute CVD risk}

Overall, $6.6 \%$ (95\% CI 5.4 to 7.8 ) of women and $15.4 \%$ $(13.9 \%$ to $16.9 \%)$ of men aged $45-74$ were considered to be at high absolute risk of a primary CVD event (figure 3 and table 2). A greater proportion of men than women were determined to be at high risk based on clinical

Table 1 Means, medians and IQR for continuous CVD risk factors in Australian population aged 45-74 years, by sex

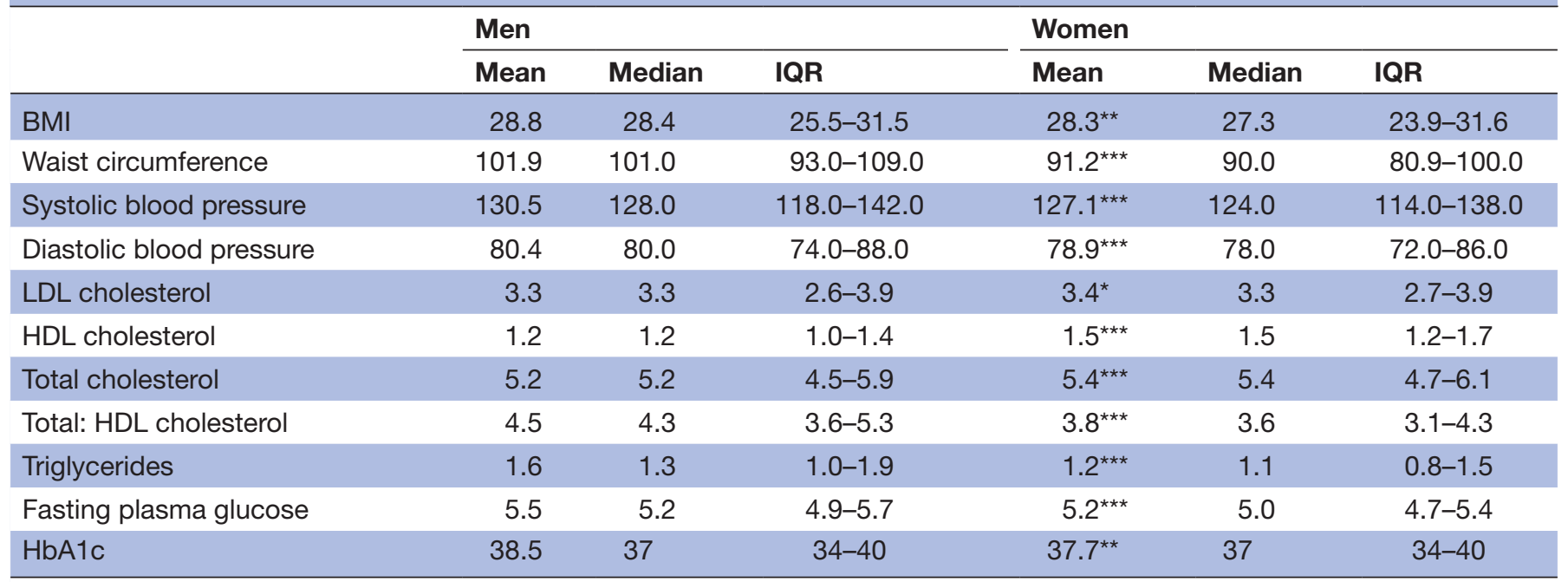

BMI, waist circumference, systolic blood pressure and diastolic blood pressure were measured as part of the core content for the Australian Health Survey ( $n=11518$ ). Proportion of missing values: BMI: 15.9\%; waist circumference: $16.0 \%$; systolic blood pressure: $15.3 \%$; diastolic blood pressure: $15.3 \%$. LDL, HDL, total and total: HDL cholesterol, triglycerides, fasting plasma glucose and HbA1c were measured as part of the National Health Measures Survey ( $n=5253$ ). Proportion of missing values: LDL cholesterol: $20.6 \%$; HDL: $0.7 \%$; total cholesterol $0.7 \%$; total: HDL cholesterol: $0.7 \%$; triglycerides: $19.4 \%$; fasting plasma glucose: $19.4 \%$; HbA1c: $0.9 \%$. All estimates have been weighted to be representative of the Australian population living in non-very remote areas.

${ }^{\star * *}$ indicates that means are significantly different $p<0.001,{ }^{* *} p<0.01,{ }^{*} p<0.05$.

BMI, body mass index; CVD, cardiovascular disease; HbA1c, haemoglobin A1c; HDL, high-density lipoprotein; LDL, low-density lipoprotein. 


\begin{tabular}{|c|c|c|c|c|c|c|}
\hline & Men \% & Women \% & $\begin{array}{l}\text { Prevalence } \\
\text { difference }\end{array}$ & $\begin{array}{l}\text { Prevalence } \\
\text { Ratio }\end{array}$ & & \\
\hline \multicolumn{7}{|l|}{$\overline{\mathrm{BMI}}$} \\
\hline Underweight/normal & 21.0 & 33.2 & $12.1(9.8,14.5)$ & $1.58(1.44,1.73)$ & & $\rightarrow$ \\
\hline Overweight & 43.9 & 33.3 & $-10.6(-12.9,-8.3)$ & $0.76(0.71,0.81)$ & a & \\
\hline Obese & 35.1 & 33.6 & $-1.5(-3.9,0.8)$ & $0.96(0.89,1.02)$ & 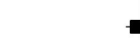 & 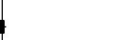 \\
\hline \multicolumn{7}{|l|}{ Waist circumference } \\
\hline Not at risk & 25.8 & 22.3 & $-3.5(-5.6,-1.5)$ & $0.86(0.79,0.94)$ & $=$ & \\
\hline At risk & 74.2 & 77.7 & $3.5(1.5,5.6)$ & $1.05(1.02,1.08)$ & & 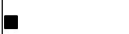 \\
\hline \multicolumn{7}{|l|}{ Physical activity } \\
\hline High & 11.2 & 8.2 & $-3.0(-4.5,-1.5)$ & $0.73(0.63,0.85)$ & $=$ & \\
\hline Moderate & 30.7 & 29.3 & $-1.4(-3.7,1.0)$ & $0.95(0.88,1.03)$ & 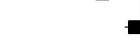 & \\
\hline Low & 35.7 & 40.6 & $4.9(2.6,7.2)$ & $1.14(1.07,1.21)$ & & $=$ \\
\hline Sedentary & 22.5 & 21.9 & $-0.5(-2.9,1.8)$ & $0.98(0.88,1.09)$ & & \\
\hline \multicolumn{7}{|l|}{ Smoking Status } \\
\hline Current & 18.4 & 14.5 & $-4.0(-5.8,-2.2)$ & $0.78(0.70,0.88)$ & $=$ & \\
\hline Former & 44.0 & 32.4 & $-11.6(-13.9,-9.3)$ & $0.74(0.69,0.78)$ & 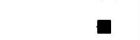 & \\
\hline Never & 37.5 & 53.1 & $15.6(13.3,17.9)$ & $1.42(1.34,1.49)$ & & $=$ \\
\hline \multicolumn{7}{|l|}{ Alcohol intake /wk } \\
\hline 0 drinks & 29.5 & 47.6 & $18.1(14.8,21.5)$ & $1.61(1.47,1.77)$ & & - \\
\hline 1-14 drinks & 41.3 & 41.3 & $0.0(-3.1,3.0)$ & $1.00(0.93,1.08)$ & t & + \\
\hline$>14$ drinks & 29.2 & 11.1 & $-18.1(-21.0,-15.1)$ & $0.38(0.32,0.45)$ & $=$ & \\
\hline \multicolumn{7}{|l|}{ Systolic BP } \\
\hline$<120 \mathrm{mmHg}$ & 30.0 & 39.7 & $9.8(7.2,12.3)$ & $1.33(1.23,1.43)$ & & $\Rightarrow$ \\
\hline $120-139 \mathrm{mmHg}$ & 42.0 & 36.5 & $-5.5(-7.8,-3.2)$ & $0.87(0.82,0.92)$ & - & \\
\hline $140-179 \mathrm{mmHg}$ & 26.8 & 22.4 & $-4.4(-6.6,-2.3)$ & $0.83(0.76,0.91)$ & $=$ & \\
\hline$\geq 180 \mathrm{mmHg}$ & 1.2 & 1.3 & $0.1(-0.5,0.8)$ & $1.12(0.68,1.86)$ & & \\
\hline \multicolumn{7}{|l|}{ Diastolic BP } \\
\hline$<90 \mathrm{mmHg}$ & 81.5 & 85.5 & $4.0(1.9,6.1)$ & $1.05(1.02,1.08)$ & & - \\
\hline $90-<110 \mathrm{mmHg}$ & 17.8 & 14.2 & $-3.6(-5.7,-1.6)$ & $0.80(0.69,0.91)$ & - & \\
\hline$\geq 110 \mathrm{mmHg}$ & 0.7 & 0.3 & $-0.4(-0.7,0.0)$ & $0.48(0.25,0.91)$ & $\longrightarrow$ & \\
\hline \multicolumn{7}{|l|}{ LDL Cholesterol } \\
\hline$<2.0 \mathrm{mmol} / \mathrm{L}$ & 8.1 & 4.4 & $-3.7(-5.5,-2.0)$ & $0.54(0.41,0.71)$ & - & \\
\hline $2.0-3.5 \mathrm{mmol} / \mathrm{L}$ & 55.4 & 55.2 & $-0.1(-4.2,4.0)$ & $1.00(0.93,1.07)$ & & + \\
\hline$>3.5 \mathrm{mmol} / \mathrm{L}$ & 36.5 & 40.4 & $3.9(-0.5,8.2)$ & $1.11(0.99,1.24)$ & & - \\
\hline \multicolumn{7}{|l|}{ HDL Cholesterol } \\
\hline$\geq 1.0 \mathrm{mmol} / \mathrm{L}$ & 80.2 & 96.5 & $16.3(14.0,18.6)$ & $1.20(1.17,1.24)$ & & - \\
\hline$<1.0 \mathrm{mmol} / \mathrm{L}$ & 19.8 & 3.5 & $-16.3(-18.6,-14.0)$ & $0.18(0.13,0.23)$ & - & \\
\hline \multicolumn{7}{|l|}{ Total Cholesterol } \\
\hline$<4.0 \mathrm{mmol} / \mathrm{L}$ & 11.0 & 6.4 & $-4.6(-6.5,-2.7)$ & $0.58(0.46,0.73)$ & - & \\
\hline $4.0-5.5 \mathrm{mmol} / \mathrm{L}$ & 57.2 & 52.9 & $-4.3(-8.0,-0.5)$ & $0.93(0.86,0.99)$ & $=$ & \\
\hline $5.6-7.5 \mathrm{mmol} / \mathrm{L}$ & 30.6 & 38.4 & $7.8(3.7,11.9)$ & $1.25(1.11,1.41)$ & & $\rightarrow$ \\
\hline$>7.5 \mathrm{mmol} / \mathrm{L}$ & 1.2 & 2.3 & $1.1(0.3,1.9)$ & $1.88(1.14,3.08)$ & & 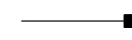 \\
\hline \multicolumn{7}{|l|}{ Total:HDL Cholesterol } \\
\hline$<4.5$ & 55.1 & 79.2 & $24.1(20.1,28.0)$ & $1.44(1.35,1.53)$ & & $\rightarrow$ \\
\hline $4.5-<6.0$ & 32.3 & 17.2 & $-15.0(-18.7,-11.3)$ & $0.53(0.46,0.62)$ & $=$ & \\
\hline$\geq 6.0$ & 12.6 & 3.5 & $-9.1(-11.5,-6.6)$ & $0.28(0.19,0.41)$ & $=$ & \\
\hline \multicolumn{7}{|l|}{ Triglycerides } \\
\hline$<1.7 \mathrm{mmol} / \mathrm{L}$ & 65.2 & 80.2 & $15.0(11.3,18.8)$ & $1.23(1.16,1.30)$ & & $=$ \\
\hline $1.7-2.0 \mathrm{mmol} / \mathrm{L}$ & 14.4 & 10.6 & $-3.7(-6.9,-0.6)$ & $0.74(0.57,0.95)$ & $\rightarrow$ & \\
\hline$>2.0 \mathrm{mmol} / \mathrm{L}$ & 20.5 & 9.2 & $-11.3(-14.2,-8.4)$ & $0.45(0.37,0.55)$ & $=$ & \\
\hline Diabetes & 12.5 & 6.8 & $-5.7(-7.7,-3.7)$ & $0.55(0.44,0.67)$ & - & \\
\hline Diabetes microalbuminuria & 3.5 & 0.9 & $-2.6(-3.5,-1.6)$ & $0.26(0.17,0.42)$ & - & \\
\hline Chronic kidney disease & 0.6 & 0.3 & $-0.3(-0.7,0.1)$ & $0.53(0.22,1.23)$ & $\longrightarrow$ & - \\
\hline
\end{tabular}

Figure 2 Age-adjusted prevalence, prevalence difference and prevalence ratios (and 95\% Cl) for CVD risk factors for the population aged 45-74 years for women versus men. Prevalence differences and prevalence ratios compare women to men. The prevalence ratio is plotted. BMI, waist circumference, systolic blood pressure and diastolic blood pressure were measured as part of the core content for the Australian Health Survey $(n=11518)$. Proportion of missing values: BMI: 15.9\%; waist circumference: $16.0 \%$; systolic blood pressure: $15.3 \%$; diastolic blood pressure: $15.3 \%$. LDL, HDL, total and total: HDL cholesterol, triglycerides, fasting plasma glucose, $\mathrm{HbA} 1 \mathrm{c}$, diabetes, diabetes with microalbuminuria and chronic kidney disease were measured as part of the National Health Measures Survey $(n=5253)$. Proportion of missing values: LDL cholesterol: $20.6 \%$; HDL: $0.7 \%$; total cholesterol 0.7\%; total: HDL cholesterol: 0.7\%; triglycerides: 19.4\%; fasting plasma glucose: 19.4\%; HbA1c: $0.9 \%$; diabetes and diabetes with microalbuminuria: $0.9 \%$; chronic kidney disease: $0.8 \%$. All estimates have been weighted to be representative of the Australian population living in non-very remote areas. An at-risk waist circumference is defined as $\geq 80 \mathrm{~cm}$ for women and $\geq 94 \mathrm{~cm}$ for men. BMI, body mass index; BP, blood pressure; CVD, cardiovascular disease; HbA1c, haemoglobinA1c; HDL, high-density lipoprotein; LDL, low-density lipoprotein. 


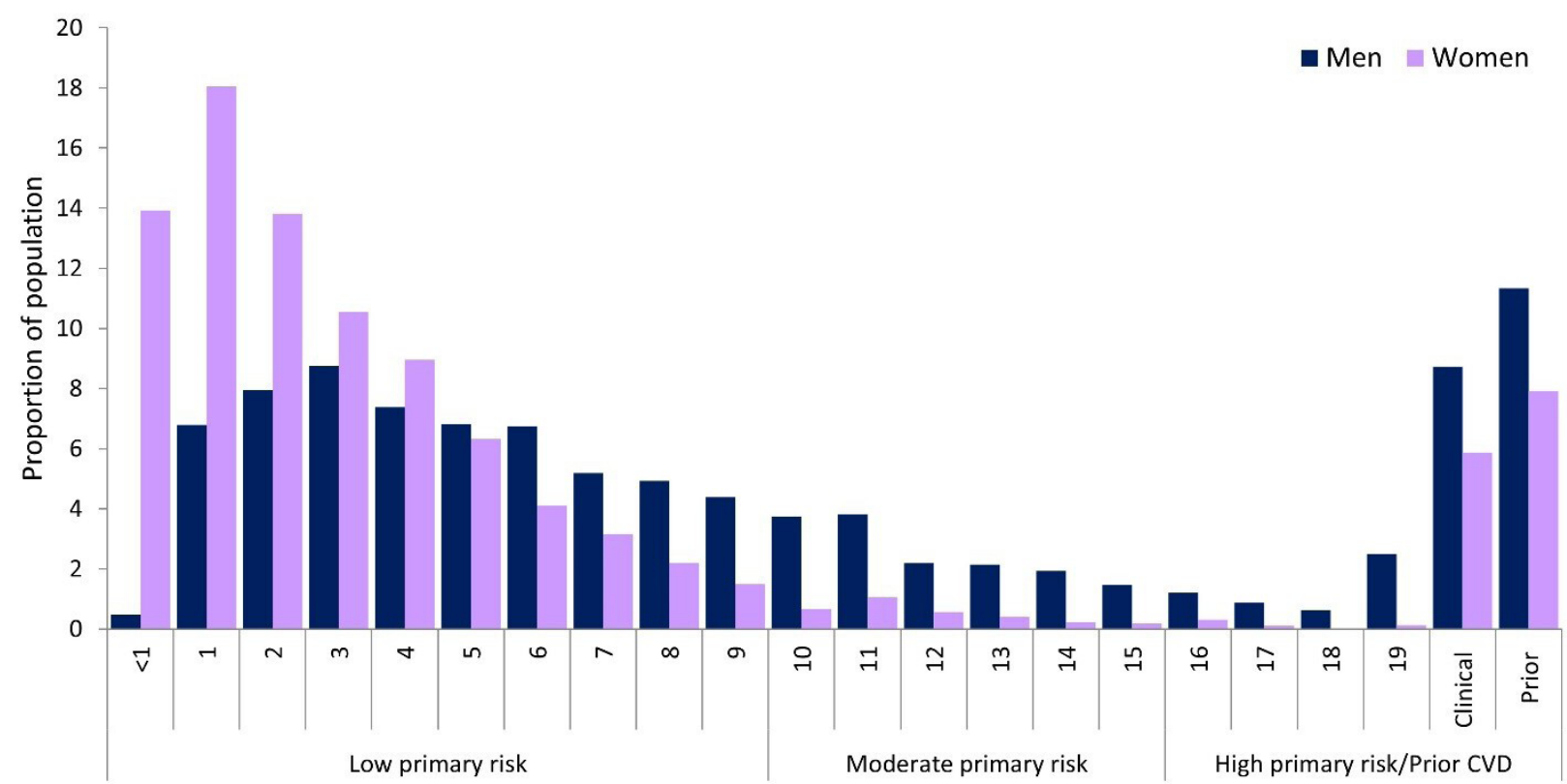

5-Year Absolute CVD Risk

Figure 3 Estimated distribution of 5 year absolute CVD risk, including clinically high risk and prior CVD, among the Australian population aged $45-74$ years, by sex. Estimates are based on 4833 people who participated in the National Health Measures Survey with no missing data on variables needed to calculate absolute CVD risk. All estimates have been weighted to be representative of the Australian population living in non-very remote areas. CVD, cardiovascular disease.

criteria $(8.7 \%$ vs $5.9 \%)$, however, among those at high absolute CVD risk, a greater proportion of women than men were so classified based on clinical criteria $(89.4 \%$ of women compared with $56.5 \%$ of men).

Overall, $2.9 \%$ (95\% CI 2.2 to 3.7 ) of women and $13.8 \%$ (11.5 to 16.1) of men were at moderate risk of a primary CVD event, and $82.6 \%$ (80.8 to 84.3) of women and $59.4 \%$ (57.0 to 61.9) of men were at low primary risk. Among people aged $\geq 18$ years, women continued to have a more favourable profile relative to men (online supplemental figure 5), (online supplemental table 5).

\section{Blood pressure and cholesterol assessment}

The large majority of the population $(88.1 \%$ of men and $88.3 \%$ of women) reported having both their blood pressure and cholesterol assessed in the last 2 and 5 years, respectively (table 3). After adjusting for age, region of residence, country of birth and education, similar proportions of men and women in the population (aged 45-74 years) received both checks (overall age-adjusted PR: $1.00,95 \%$ CI 0.96 to 1.05 , PD: $0.27 \%, 95 \%$ CI -0.34 to 0.40 ). However, among those with prior CVD, an additional $5.4 \%$ (95\% CI -0.1 to 10.9 ) of women compared with men had received both checks (PR 1.06, 95\% CI 1.00 to 1.12) (table 3).

\section{Medication use}

Overall, the proportion of men and women without prior CVD using blood pressure-lowering and lipid-lowering medications increased as absolute CVD risk increased but remained low (figure 4 and online supplemental Table S6), such that $21.0 \%$ (9.4 to 32.6) of women and $24.0 \%$ (16.3 to 31.7 ) of men at high absolute primary risk of a CVD event were using both recommended medications (adjusted PR: $0.93,95 \%$ CI 0.49 to 1.78 ; table 4). Among Australians with prior atherosclerotic CVD, 28.1\% (20.9

Table 2 Estimated proportions (with 95\% Cls) and number of individuals in the Australian population aged 45-74 years in each CVD risk category, by sex

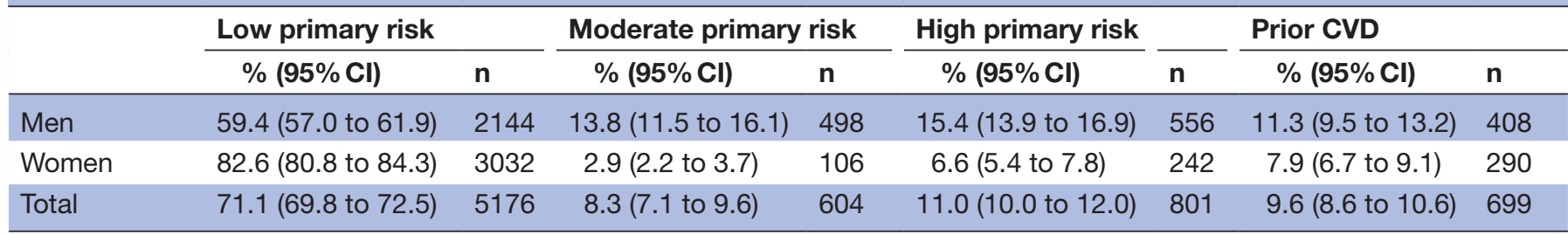

$\mathrm{n}=$ estimated number, in thousands, of persons in each category in the Australian population. Weighting and missing values mean that numbers do not always sum to totals. Estimates are based on 4833 people who participated in the National Health Measures Survey and had no missing data on variables used to estimate absolute CVD risk. All estimates have been weighted to be representative of the Australian population living in non-very remote areas.

CVD, cardiovascular disease. 
Table 3 Relative and absolute differences in prevalence of women compared with men aged 45-74 years reporting both blood pressure and cholesterol assessments in the previous 2 and 5 years respectively

\begin{tabular}{|c|c|c|c|}
\hline & \multirow{2}{*}{$\begin{array}{l}\text { Age-adjusted prevalence both } \\
\text { assessments } \\
\%(95 \% \mathrm{Cl})\end{array}$} & \multicolumn{2}{|l|}{ Multivariable adjusted $^{*}$} \\
\hline & & $\begin{array}{l}\text { Prevalence difference } \\
(95 \% \mathrm{Cl})\end{array}$ & $\begin{array}{l}\text { Prevalence ratio } \\
(95 \% \mathrm{Cl})\end{array}$ \\
\hline \multicolumn{4}{|c|}{ Low primary risk } \\
\hline Men & 86.5 (82.7 to 90.2 ) & 0.0 & 1.00 \\
\hline Women & 87.1 (84.6 to 89.7 ) & $1.3(-3.1$ to 5.8$)$ & 1.02 (0.97 to 1.07$)$ \\
\hline \multicolumn{4}{|c|}{ Moderate primary risk } \\
\hline Women & 87.9 (76.2 to 99.7$)$ & $-2.1(-16.4$ to 12.2$)$ & 0.98 (0.83 to 1.15$)$ \\
\hline \multicolumn{4}{|c|}{ High absolute risk } \\
\hline Men & 90.3 (83.8 to 96.7 ) & 0.0 & 1.00 \\
\hline Women & 84.6 (74.6 to 94.6$)$ & $-5.2(-15.6$ to 5.2$)$ & 0.94 (0.84 to 1.06$)$ \\
\hline \multicolumn{4}{|l|}{ Prior CVD } \\
\hline
\end{tabular}

Estimates are based on 2875 people who participated in the National Health Survey and the National Health Measures Survey who had enough information to estimate absolute CVD risk. All estimates have been weighted to be representative of the Australian population living in non-very remote areas.

${ }^{*}$ Adjusted for age, country of birth, highest level of education and region of residence.

CVD, cardiovascular disease.

to 35.4 ) of women and $41.6 \%$ (34.7 to 48.5$)$ of men were using all three of blood pressure lowering, lipid-lowering medications and antithrombotic medications (adjusted PR: $0.55,95 \%$ CI 0.31 to 0.96 ; table 4$)$. Of $16.8 \%(10.5$ to 23.2$)$ of women and $11.0 \%(7.0,15.1)$ of men with atherosclerotic CVD were not receiving any of these medications. The prevalence of and sex differences in CVD medication use was not materially different when prior CVD was restricted to ischaemic heart disease only.

\section{DISCUSSION}

Using nationally representative Australian data, this study demonstrates generally more favourable profiles for women than men for CVD risk factors, absolute risk of a primary CVD event and the prevalence of prior CVD. Around one-quarter of those at high absolute risk and less than half of those with prior CVD were receiving guideline-recommended medications. While there was no observed difference by sex in treatment of high primary

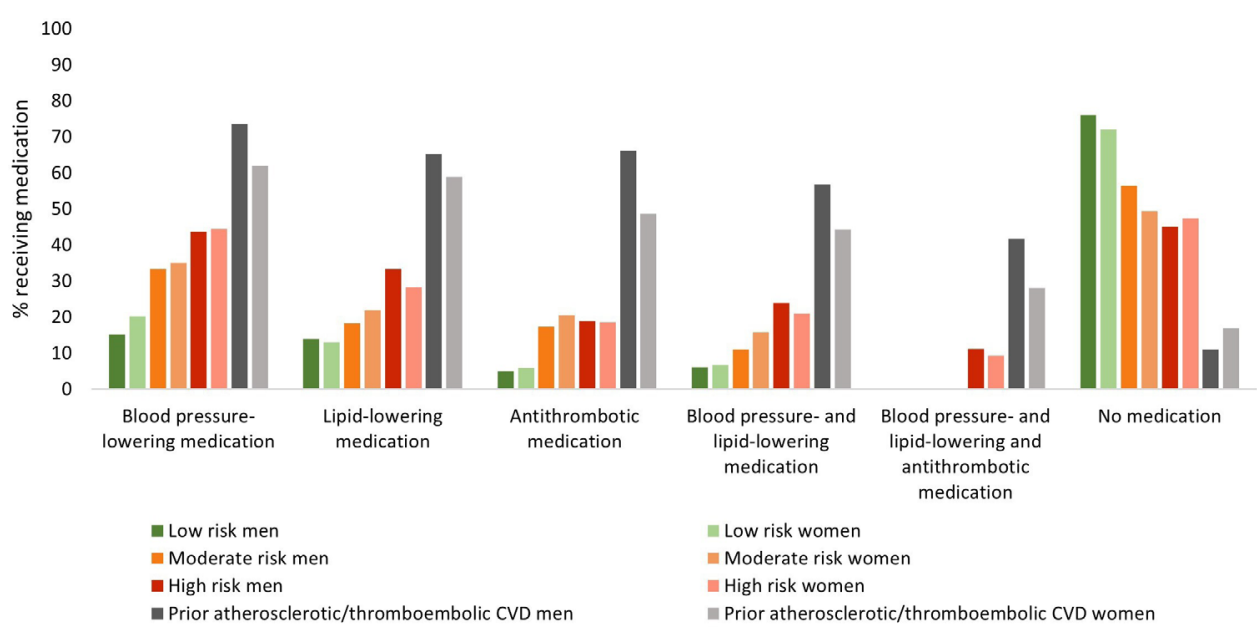

Figure 4 Estimated proportions in the Australian population aged 45-74 years using cardiovascular disease medications for those at low, moderate and high primary CVD risk and those with prior atherosclerotic/thromboembolic CVD, by sex. Estimates are based on 2847 people who participated in the National Health Survey and the National Health Measures Survey and had enough information to estimate absolute CVD risk. All estimates have been weighted to be representative of the Australian population living in non-very remote areas. No medication refers to no blood pressure-lowering, lipid-lowering or antithrombotic medications. Proportions receiving blood pressure-lowering and lipid-lowering and antithrombotic medication among those at low or moderate absolute risk of primary CVD have been suppressed due to small cell sizes. CVD, cardiovascular disease. 
risk, women with prior CVD were around half as likely as men to be using recommended medications.

We find that, compared with men, Australian women have a lower average waist circumference and BMI, are more likely to be of normal weight or underweight, are less likely to be overweight and have similar prevalences of obesity. Compared with men, women have lower mean fasting blood glucose levels and around half the prevalence of diabetes; somewhat lower levels of systolic and diastolic blood pressure and raised blood pressure overall; higher mean LDL, HDL and total cholesterol levels; lower prevalence of daily smoking; greater level of sedentary behaviours and lower level of physical activity. These findings are generally consistent with those from other high-income countries. ${ }^{19-22}$

The exact reasons for the sex differences in risk factors observed here are not known and are likely to have multiple biological and sociocultural contributors. Higher BMI and waist circumference in men are likely to contribute to higher blood pressure, fasting plasma glucose and cholesterol levels and diabetes prevalence. Smoking is also known to increase blood pressure. ${ }^{23} 24$ Current evidence indicates that sex differentials in coronary heart disease risk are unlikely to be explained by levels of oestrogen or progesterone. The risk of death from coronary heart disease in men is higher than that of women throughout the life span, with no inflection apparent in the age-coronary heart disease mortality curve in women at the time of the menopause, ${ }^{25}$ despite large differences in premenopausal versus postmenopausal endogenous oestradiol and progesterone levels, nor do postmenopausal exogenous oestrogens appear to influence coronary heart disease risk. ${ }^{26}$

A high proportion of the Australian population and equal proportions of men and women have had their blood pressure and cholesterol assessed within the appropriate minimum window for the general population. This suggests that primary care and other health professionals are carrying out appropriate checks and patients are receptive to these, but that there is room for improvement to achieve complete coverage.

The lack of any observed sex differences in guidelinerecommended treatment of high primary risk has not, to our knowledge, been reported before and is reassuring although overall treatment is low. The finding that women with prior CVD are less likely than men to be receiving guideline-recommended treatment is consistent with findings from non-representative studies from Australia and the USA. ${ }^{27-29}$ Reasons for this remain unclear ${ }^{27}$ but are likely to be multifactorial, reflecting a variety of system-related, physician-related and patient-related factors. Differences in perception of CVD risk in women, including the idea that heart disease primarily affects men-either by medical professionals or by women themselves, may result in sexrelated differences in application of clinical guidelines and less judicious management of CVD among women compared with men. ${ }^{28} 29$ Other manifestations of unconscious bias cannot be excluded. There is also the possibility that CVD in women has different clinical features, although these are unlikely to fully explain the differences treatment of prior CVD as all patients with atherosclerotic and/or thromboembolic CVD are indicated for blood pressurelowering and lipid-lowering and antithrombotic medications, ${ }^{16}{ }^{17}$ and sex differences remained after restricting the sample to those with ischaemic heart disease. Finally, on average, women tend to develop CVD at an older age than men and this is also likely to be accompanied by higher levels of comorbidity and frailty, which could also influence treatment decisions.

This paper is the first, to our knowledge, to compare men and women comprehensively in terms of data on CVD risk factors, absolute risk, assessment and treatment. Such data are useful in quantifying opportunities for prevention and reduction of disparities across the

Table 4 Relative and absolute differences in prevalence of individuals aged 45-74 years with high absolute primary CVD risk or prior atherosclerotic CVD using guideline-recommended medications, by sex

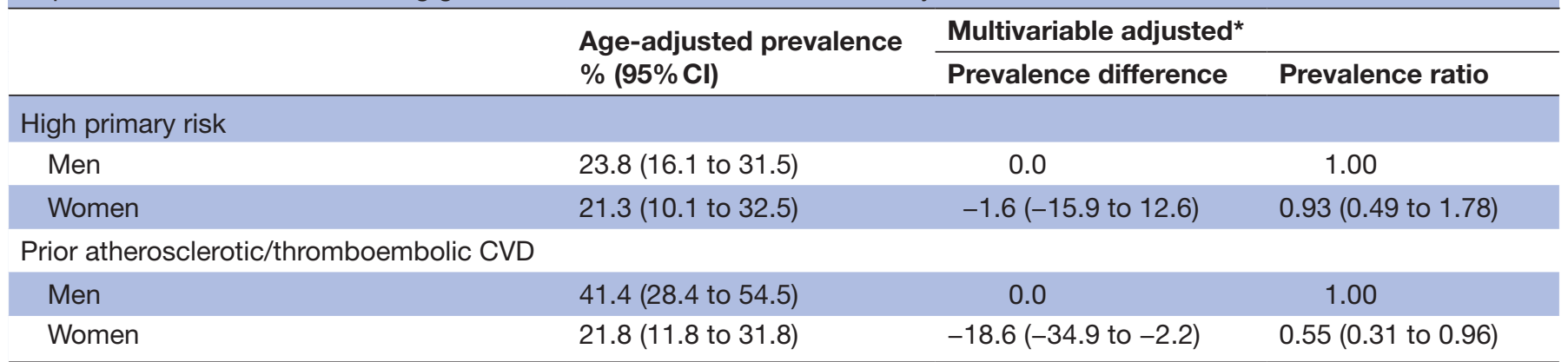

Estimates are based on 341 people high absolute CVD risk and 228 people with prior atherosclerotic CVD who participated in both the National Health Survey and the National Health Measures Survey who had enough information to estimate absolute CVD risk. All estimates have been weighted to be representative of the Australian population living in non-very remote areas. Recommended medication is blood pressure-lowering and lipid-lowering medication for those at high primary risk, and blood pressure-lowering and lipid-lowering medication, and antithrombotic medication for people with prior CVD.

${ }^{*}$ Adjusted for age, country of birth, highest level of education and region of residence.

CVD, cardiovascular disease. 
CVD continuum. This study used high-quality nationally representative self-reported and biomedical data. It had data on a one-off assessment of blood pressure and lipid levels and on pharmacological treatment to reduce blood pressure and lipid levels. It was neither able to capture behavioural or lifestyle interventions nor were data available on the reasons for a lack of treatment for people with existing CVD or with high absolute risk. Prior CVD was defined broadly from self-reported information. However, the observed finding of substantially greater treatment levels in men compared with women persisted when narrower definitions of CVD were used.

CVD is highly preventable and optimal use of current treatments is able to more than halve risk of future events. ${ }^{45}$ Substantial proportions of the Australian population are at high absolute CVD risk and the majority of those at high absolute risk and with prior CVD is not receiving basic recommended pharmacotherapy. The marked under-treatment of women in secondary CVD prevention is particular cause for concern. More than half a million Australian women are currently living with CVD, including approximately 200000 women living with ischaemic heart disease and a similar number with a history of stroke. ${ }^{30}$ Hence, there are substantial opportunities to continue to prevent premature morbidity and mortality from CVD, through improving implementation of risk assessment and management practices in the population. There is a clear need to ensure adequate treatment across the board, with a particular focus on ensuring those with prior CVD, including women, have the opportunity to receive best-practice care, including preventive medications.

Contributors EB and RK conceived the idea for the study. JW conducted the analyses and GJ provided statistical advice. EB, JW, MM and EP drafted the manuscript. All authors read and approved the manuscript.

Funding This research was supported by a NSW CVRN Women and Heart Disease Grant (Award 101692) from the National Heart Foundation of Australia (N.S.W. Division). EB is supported by the National Health and Medical Research Council of Australia (1042717).

Competing interests None declared.

Patient consent for publication Not required.

Ethics approval Ethics approval for this study was granted by the Australian National University Human Research Ethics Committee (2014/208).

Provenance and peer review Not commissioned; externally peer reviewed.

Data availability statement The data analysed for this study is available from the Australian Bureau of Statistics. Information regarding access is available here: http://abs.gov.au/websitedbs/D3310114.nsf/home/About+CURF+Microdata.

Supplemental material This content has been supplied by the author(s). It has not been vetted by BMJ Publishing Group Limited (BMJ) and may not have been peer-reviewed. Any opinions or recommendations discussed are solely those of the author(s) and are not endorsed by BMJ. BMJ disclaims all liability and responsibility arising from any reliance placed on the content. Where the content includes any translated material, BMJ does not warrant the accuracy and reliability of the translations (including but not limited to local regulations, clinical guidelines, terminology, drug names and drug dosages), and is not responsible for any error and/or omissions arising from translation and adaptation or otherwise.

Open access This is an open access article distributed in accordance with the Creative Commons Attribution Non Commercial (CC BY-NC 4.0) license, which permits others to distribute, remix, adapt, build upon this work non-commercially, and license their derivative works on different terms, provided the original work is properly cited, appropriate credit is given, any changes made indicated, and the use is non-commercial. See: http://creativecommons.org/licenses/by-nc/4.0/.

\section{ORCID iDs}

Emily Banks http://orcid.org/0000-0002-4617-1302

Jennifer Welsh http://orcid.org/0000-0003-4415-5920

Grace Joshy http://orcid.org/0000-0002-0718-6368

Melonie Martin http://orcid.org/0000-0002-9408-3470

Ellie Paige http://orcid.org/0000-0003-0855-9872

Rosemary J Korda http://orcid.org/0000-0002-9390-2171

\section{REFERENCES}

$1 \mathrm{Kyu} \mathrm{HH}$, Abate D, Abate KH, et al. Global, regional, and national disability-adjusted life-years (DALYs) for 359 diseases and injuries and healthy life expectancy (HALE) for 195 countries and territories, 1990-2017: a systematic analysis for the global burden of disease study 2017. The Lancet 2018;392:1859-922.

2 Foreman KJ, Marquez N, Dolgert A. Forecasting life expectancy, years of life lost, and all-cause and cause-specific mortality for 250 causes of death: reference and alternative scenarios for 2016-40 for 195 countries and territories. The Lancet 2018;392:2052-90.

3 Australian Institute of Health and Welfare. Cardiovascular disease snapshot. Canberra: AlHW, 2018.

4 Blood Pressure Lowering Treatment Trialists' Collaboration. Blood pressure-lowering treatment based on cardiovascular risk: a metaanalysis of individual patient data. The Lancet 2014;384:591-8.

5 Cholesterol Treatment Trialists' (CTT) Collaborators. The effects of lowering LDL cholesterol with statin therapy in people at low risk of vascular disease: meta-analysis of individual data from 27 randomised trials. The Lancet 2012;380:581-90.

6 National Vascular Disease Prevention Alliance. Guidelines for the management of absolute cardiovascular disease risk. Canberra: NVDPA, 2012.

7 Australian Institute of Health and Welfare. Cardiovascular disease: Australian facts 2011. cat no CVD 53. Canberra: AlHW, 2011.

8 Nichols MPK, Herbert J, Alston L, et al. Australian heart disease statistics 2015. National Heart Foundation of Australia 2016.

9 Australian Institute of Health and Welfare. Women and heart disease: cardiovascular profile of women in Australia. Cardiovascular disease series No. 33. Cat. no. CVD 49. Canberra AlHW; 2010.

10 Kuhn L, Page K, Rolley JX, et al. Effect of patient sex on triage for ischaemic heart disease and treatment onset times: a retrospective analysis of Australian emergency department data. Int Emerg Nurs 2014;22:88-93.

11 Hyun KK, Redfern J, Patel A, et al. Gender inequalities in cardiovascular risk factor assessment and management in primary healthcare. Heart 2017;103:492-8.

12 Australian Bureau of Statistics. Australian Health Survey 2011-12, expanded Confidentialised Unit Record File (CURF), Remote Access Data Laboratory (RADL). Canberra: Findings Based on Use of ABS Microdata, 2011.

13 Australian Bureau of Statistics. Australian Health Survey: Users' Guide, 2011-13. cat. No. 4363.0.55.001. Canberra: ABS, 2013. Available: http://www.abs.gov.au/ausstats/abs@.nsf/Latestproducts/ 4363.0.55.001Main\%20Features12011-13?opendocument\& tabname $=$ Summary \&prodno $=4363.0 .55 .001 \&$ issue $=2011-13 \&$ num $=\&$ view $=$

14 The Royal Australian College of General Practitioners. Guidelines for preventive activities in general practice. 2016. 9th edition. East Melbourne, Victoria: RACGP, 2016.

15 World Health Organization Collaborating Centre for Drug Statistics Methodology. ATC/DDD Index 2018: WHO, 2018. Available: https:// www.whocc.no/atc_ddd_index/

16 National Heart Foundation of Australia and the Cardiac Society of Australia and New Zealand. Reducing risk in heart disease: an expert guide to clinical practice for secondary prevention of coronary heart disease. Melbourne, Australia: National Heart Foundation of Australia, 2012

17 National Stroke Foundation. Clinical guidelines for stroke management 2017. Melbourne, Australia 2017.

18 Australian Bureau of Statistics. Weighting, benchmarks and estimation procedures. Australian health survey: users' guide, 201113. cat. No. 4363.0.55.001. Canberra: ABS, 2013. Available: http:// 
www.abs.gov.au/ausstats/abs@.nsf/Lookup/CD71A707B04A8F5B CA257BBB0014CBEC?opendocument

19 World Health Organization. Prevalence of obesity among adults, BMI $\geq 30$, age-standardized. Estimates by World Bank Income Group Geneva WHO, 2017. Available: http://apps.who.int/gho/data/view. main.WB2480A?lang=en

20 Zhou B, Bentham J, Di Cesare M, et al. Worldwide trends in blood pressure from 1975 to 2015: a pooled analysis of 1479 populationbased measurement studies with 19.1 million participants. The Lancet 2017;389:37-55.

21 Farzadfar F, Finucane MM, Danaei G. National, regional, and global trends in serum total cholesterol since 1980: systematic analysis of health examination surveys and epidemiological studies with 321 country-years and 3.0 million participants. The Lancet 2011;377:578-86.

22 Giovino GA, Mirza SA, Samet JM, et al. Tobacco use in 3 billion individuals from 16 countries: an analysis of nationally representative cross-sectional household surveys. The Lancet 2012;380:668-79.

23 Bowman TS, Gaziano JM, Buring JE, et al. A prospective study of cigarette smoking and risk of incident hypertension in women. J Am Coll Cardiol 2007;50:2085-92.
24 Halperin RO, Michael Gaziano J, Sesso HD. Smoking and the risk of incident hypertension in middle-aged and older men. Am J Hypertens 2008;21:148-52.

25 Woodward M. Cardiovascular disease and the female disadvantage. Int J Environ Res Public Health 2019;16:1165.

26 Medicines and Healthcare Products Regulatory Agency. HormoneReplacement therapy: safety update London MHRA 2007.

27 Lee CMY, Mnatzaganian G, Woodward M, et al. Sex disparities in the management of coronary heart disease in general practices in Australia. Heart 2019;105:1898-1904.

28 Turnbull F, Arima H, Heeley E, et al. Gender disparities in the assessment and management of cardiovascular risk in primary care: the AusHEART study. Eur J Cardiovasc Prev Rehabil 2011;18:498-503.

29 Manteuffel M, Williams S, Chen W, et al. Influence of patient sex and gender on medication use, adherence, and prescribing alignment with guidelines. J Womens Health 2014;23:112-9.

30 Australian Institute of Health and Welfare. Cardiovascular disease in Australian women - a snapshot of national statistics. Canberra: AlHW, 2019. 